\title{
A density functional approach to depletion interaction
}

\author{
José A Cuesta and Yuri Martínez-Ratón \\ Grupo Interdisciplinar de Sistemas Complicados, Departamento de Matemáticas, Universidad \\ Carlos III de Madrid, 28911-Leganés, Madrid, Spain
}

\begin{abstract}
In this article we introduce depletion in asymmetric mixtures within the context of density functional theory. As in the definition of the interaction potentials, it is convenient to work in a semi-grand ensemble at constant chemical potential of the small particles. The free-energy functional can then be mapped onto that of a one-component effective fluid. This method is applied to the fluid of parallel hard cubes, which is studied in the limit of infinite asymmetry. The phase behaviour of this fluid is shown and discussed in terms of the general phase behaviour of additive mixtures of hard particles.
\end{abstract}

\section{Introduction}

Since Biben and Hansen showed evidence that, contrary to what was then believed, a binary mixture of hard spheres can phase separate [1], we have come in a decade to a coherent picture of this phenomenon in terms of its basic mechanism: depletion. This was first suggested long ago by Asakura and Oosawa [2] as an effective attraction of a purely entropic nature: there is a global gain in free volume if two large particles whose surfaces are separated by less than the size of a small one come closer to each other. Consequently there is a gain in entropy that in some cases compensates for the loss produced by the clustering of the large particles. This simple picture can be approximately translated into an effective pairwise attraction which is basically composed of a narrow (the range is the small-particle diameter) and deep (the depth is determined by the density of the small particles) well. Although this is essentially correct, we now know that such a potential exhibits a richer structure, even being weakly repulsive at certain distances [3]. The reason is that the fluid of small particles behaves in between the big ones as a fluid confined between walls.

The most interesting thing about the depletion interaction is that, properly accounted for, it contains all the information about the phase behaviour of the mixture. In other words, the effective one-component fluid formed by the large spheres with the depletion potential added to their direct interactions behaves in exactly the same way as the mixture [4-8]. (Of course if the mixture is not sufficiently asymmetric the depletion pair potential is not enough, but we can correct it with triplet, quadruplet, etc, potentials; this point will be made clear later.) As a consequence, an asymmetric binary mixture shares the phase behaviour with colloids interacting through 'narrow and deep' pair potentials. It is now well known that the latter can lose the liquid phase, widening the fluid-solid coexistence region at low temperatures, and even exhibit an 'exotic' isostructural solid-solid transition $[9,10]$. In terms of a mixture, a fluid-solid transition means fluid-solid demixing, i.e. a phase separation in which the phase rich in large particles is a solid; and a solid-solid transition means that in both phases the large 
particles form a solid, but in the one richer in large particles it is very highly packed. Results of simulations and analyses performed on hard spheres [5-7] and parallel hard cubes [8] show that this is indeed the demixing scenario for sufficiently asymmetric mixtures.

Once we have the pair potential of a fluid system it is clear how to carry out simulations or how to apply integral equations, for instance; but the knowledge of the potential is of little interest as regards devising a density functional theory. Very recently we have incorporated the depletion picture into one such theory and have applied it to study the binary mixture of parallel hard cubes [8]. We are going to discuss in this contribution how to achieve this in general, and will describe the outline of its application to that system. The results illustrate the qualitative behaviour of very asymmetric binary mixtures, but our main purpose is to emphasize the generality of this formalism.

The organization of the paper is as follows: we express depletion in terms of both effective interaction potentials and density functional theory in section 2; we then apply it to parallel hard cubes in the limit of infinite asymmetry in section 3; and finally we draw some conclusions in section 4 .

\section{Depletion}

\subsection{Definition}

Let us consider a binary mixture of two species of particles, large (L) and small (S), in a cavity of volume $V$ and temperature $T$. Suppose there are a fixed number of large (solute) particles, $N_{\mathrm{L}}$, and that the small (solvent) ones are in contact with a reservoir of particles at chemical potential $\mu_{\mathrm{S}}$. If we denote the interaction energy between the particles of species $\alpha$ and $\gamma$ (in units of $k T$ ) as $\phi_{\alpha \gamma}$, then the partition function of this semi-grand ensemble is given by

$$
Z=\operatorname{tr}_{\mathrm{L}} \operatorname{Tr}_{\mathrm{S}} \exp \left\{-\phi_{\mathrm{LL}}-\phi_{\mathrm{LS}}-\phi_{\mathrm{SS}}\right\}
$$

where

$\operatorname{tr}_{\mathrm{L}}=\frac{1}{N_{\mathrm{L}} ! \mathcal{V}_{\mathrm{L}}^{N_{\mathrm{L}}}} \int_{V} \mathrm{~d} \boldsymbol{R}_{1} \cdots \int_{V} \mathrm{~d} \boldsymbol{R}_{N_{\mathrm{L}}} \quad \operatorname{Tr}_{\mathrm{S}}=\sum_{N_{\mathrm{S}}=1}^{\infty} \frac{z^{N_{\mathrm{S}}}}{N_{\mathrm{S}} !} \int_{V} \mathrm{~d} \boldsymbol{r}_{1} \cdots \int_{V} \mathrm{~d} \boldsymbol{r}_{N_{\mathrm{S}}}$.

Here $z=\mathrm{e}^{\beta \mu_{\mathrm{S}}} / \mathcal{V}_{\mathrm{S}}$ is the solvent excess fugacity, $\mathcal{V}_{\mathrm{L}, \mathrm{S}}$ the solute/solvent thermal volume, and $\boldsymbol{R}_{i}$ and $\boldsymbol{r}_{j}$ denote the positions of the large and small particles, respectively.

Let us define

$$
\exp (-\Omega) \equiv \operatorname{Tr}_{\mathrm{S}} \exp \left\{-\phi_{\mathrm{LS}}-\phi_{\mathrm{SS}}\right\}
$$

It is clear that $\Omega$ is a function of $\left\{\boldsymbol{R}_{1}, \ldots, \boldsymbol{R}_{N_{\mathrm{L}}}\right\}, z, V$, and $T$. In terms of this function,

$$
Z=\operatorname{tr}_{\mathrm{L}} \exp \left\{-\phi_{\mathrm{LL}}-\Omega\right\} \equiv \operatorname{tr}_{\mathrm{L}} \exp \left(-\phi_{\mathrm{L}}^{\mathrm{eff}}\right) .
$$

In other words, $\Omega$ can be interpreted as an effective energy of interaction between the solute particles (in units of $k T$ ) mediated by the solvent. This is depletion.

From the diagrammatic expansion of $\Omega[5]$ it can be shown that

$\Omega=-V p_{0}(z)+N_{\mathrm{L}} \omega_{1}(z)+\sum_{i<j}^{N_{\mathrm{L}}} \omega_{2}\left(\boldsymbol{R}_{i}, \boldsymbol{R}_{j} ; z\right)+\sum_{i<j<k}^{N_{\mathrm{L}}} \omega_{3}\left(\boldsymbol{R}_{i}, \boldsymbol{R}_{j}, \boldsymbol{R}_{k} ; z\right)+\cdots$

where $p_{0}(z)$ is the pressure of the pure solvent at excess fugacity $z$ and $\omega_{1}(z)$ is the work necessary to insert a single solute particle in the solvent (and thus equals the excess chemical potential of the large particles at zero density). Although these two terms contribute to the thermodynamic potentials, they are utterly irrelevant for the phase behaviour of the fluid (they 
simply add constants to the pressure and chemical potential of the effective fluid); hence they can harmlessly be ignored. The position-dependent terms $\omega_{2}, \omega_{3}$, etc, have a simple physical meaning: $\omega_{2}\left(\boldsymbol{R}_{i}, \boldsymbol{R}_{j} ; z\right)$ is the work necessary to bring two large particles already in the system from infinity to positions $\boldsymbol{R}_{i}$ and $\boldsymbol{R}_{j} ; \omega_{3}\left(\boldsymbol{R}_{i}, \boldsymbol{R}_{j}, \boldsymbol{R}_{k} ; z\right)$ is the same work for three particles minus the sum of the three pairwise interactions; and so on.

The identification of the depletion potential is useful for simulations of rather asymmetric binary mixtures, a regime in which $\omega_{2}$ is the dominant term (apart from the trivial terms $\left.-V p_{0}+N_{\mathrm{L}} \omega_{1}\right)$ and hence triplet and higher interactions can be ignored (they never vanish, though). This approximation has been successfully exploited by different authors [4-6] in simulations of binary mixtures of hard spheres. However, if one wants to construct a theory to describe such systems, the knowledge of the interaction potential is not very useful unless a perturbative scheme is adopted [7]. But let us face the same problem within density functional theory.

\subsection{The density functional framework}

In density functional theory (DFT) one wants to have a functional of the one-particle density rather than the microscopic interaction potential. This is reflected in the fact that in constructing one such functional a typical ingredient is the direct correlation function (DCF), either included directly or through some weighting function [11]. In the previous section we started off from a binary system whose interactions were known a priori. In order to construct a DFT for the depleted fluid we must have a reasonably good functional for this binary fluid. Suppose we do indeed have an expression for the Helmholtz free-energy functional:

$$
F\left[\rho_{\mathrm{L}}, \rho_{\mathrm{S}}\right]=k T \sum_{i=\mathrm{L}, \mathrm{S}} \int \mathrm{d} \boldsymbol{r} \rho_{i}(\boldsymbol{r})\left[\ln \mathcal{V}_{i} \rho_{i}(\boldsymbol{r})-1\right]+F^{\mathrm{ex}}\left[\rho_{\mathrm{L}}, \rho_{\mathrm{S}}\right]
$$

As in the former case, adopting the depletion picture amounts to describing the system in the semi-grand ensemble in which the solvent is kept at constant chemical potential, $\mu_{\mathrm{S}}$, and fixed external field, $V_{\mathrm{S}}(\boldsymbol{r})$; we must then use the Legendre transform of our functional:

$$
\Upsilon\left[u_{\mathrm{S}}, \rho_{\mathrm{L}}\right]=F\left[\rho_{\mathrm{L}}, \rho_{\mathrm{S}}\right]-\int u_{\mathrm{S}} \rho_{\mathrm{S}} \mathrm{d} \boldsymbol{r}
$$

where $u_{\mathrm{S}}(\boldsymbol{r})=\mu_{\mathrm{S}}-V_{\mathrm{S}}(\boldsymbol{r})$, and eliminate $\rho_{\mathrm{S}}(\boldsymbol{r})$ in terms of $u_{\mathrm{S}}(\boldsymbol{r})$ and $\rho_{\mathrm{L}}(\boldsymbol{r})$ through the equilibrium condition

$$
u_{\mathrm{S}}(\boldsymbol{r})=\frac{\delta F}{\delta \rho_{\mathrm{S}}(\boldsymbol{r})}=k T \ln \mathcal{V}_{\mathrm{S}} \rho_{\mathrm{S}}(\boldsymbol{r})+\frac{\delta F^{\mathrm{ex}}}{\delta \rho_{\mathrm{S}}(\boldsymbol{r})} .
$$

From $\Upsilon$ we can separate out the ideal term

$$
\int \rho_{\mathrm{L}}\left(\ln \mathcal{V}_{\mathrm{L}} \rho_{\mathrm{L}}-1\right) \mathrm{d} \boldsymbol{r}
$$

and the excess part (the remainder). However, this excess part does not behave as the excess free energy of any other fluid, whose lower-order dependence on the density is $\mathrm{O}\left(\rho^{2}\right)$. In this case, due to the contribution of the ideal free energy of the solvent, there are also terms $\mathrm{O}(1)$ and $\mathrm{O}(\rho)$. The $\mathrm{O}(1)$ term can easily be obtained by taking $\rho_{\mathrm{L}} \rightarrow 0$ in (5) and (6). In this limit the latter equation gives us the equilibrium profile of the pure solvent fluid at the fixed chemical potential; therefore the former equation is the equilibrium grand potential of this fluid-in other words,

$$
-V p_{0}\left[u_{\mathrm{S}}\right] \text {. }
$$


For zero external field, $p_{0}\left[u_{\mathrm{S}}\right]$ reduces to the function $p_{0}(z)$ introduced in equation (4). As for the $\mathrm{O}(\rho)$ term, if we differentiate the excess part of $\Upsilon$ we find

$$
\frac{\delta F}{\delta \rho_{L}(\boldsymbol{r})}-\ln \mathcal{V}_{\mathrm{L}} \rho_{L}(\boldsymbol{r})
$$

(there is also an implicit dependence on $\rho_{\mathrm{L}}$ through $\rho_{\mathrm{S}}$, according to (6), but notice that, due precisely to this equation, $\delta \Upsilon / \delta \rho_{\mathrm{S}}=0$ ). In the limit $\rho_{\mathrm{L}} \rightarrow 0$ this expression becomes the excess chemical potential of the solute particles at infinite dilution, or, in other words, the work needed to insert a single solute particle in a pure solvent at the fixed chemical potential $\omega_{1}\left(r ;\left[u_{\mathrm{S}}\right]\right)$. Accordingly the $\mathrm{O}(\rho)$ term will simply be

$$
\int \mathrm{d} \boldsymbol{r} \rho_{\mathrm{L}}(\boldsymbol{r}) \omega_{1}\left(\boldsymbol{r} ;\left[u_{\mathrm{S}}\right]\right)
$$

In the absence of an external field this reduces to $N_{\mathrm{L}} \omega_{1}(z)$, the second position-independent term obtained in the previous section.

Collecting these two terms, we can write

$$
\Upsilon=-V p_{0}+\int \rho_{\mathrm{L}} \omega_{1} \mathrm{~d} \boldsymbol{r}+F_{\text {eff }}
$$

where now $F_{\text {eff }}$ can properly be taken as the free energy of the effective one-component depleted fluid, in which $z$ plays the role of a parameter tuning the interaction strength. Notice here the equivalence of this description to the one in terms of the partition function, as the equilibrium $\Upsilon$ is given by $-k T \ln Z$. For the sake of brevity we will henceforth use the notation

$$
\Upsilon_{0}\left[u_{\mathrm{S}}\right] \equiv-V p_{0}\left[u_{\mathrm{S}}\right]+\int \mathrm{d} \boldsymbol{r} \rho_{\mathrm{L}}(\boldsymbol{r}) \omega_{1}\left(\boldsymbol{r} ;\left[u_{\mathrm{S}}\right]\right) .
$$

According to (7) we can obtain the free-energy functional for this effective fluid for a given density profile $\rho(\boldsymbol{r})$ as

$$
F_{\text {eff }}\left[u_{\mathrm{S}}, \rho\right]=-\Upsilon_{0}\left[u_{\mathrm{S}}\right]+\Upsilon\left[u_{\mathrm{S}}, \rho\right] .
$$

We want to stress here that, regardless of the way in which we have derived $F_{\text {eff }}$, it is the true free-energy functional for the effective model defined by the (many-body) interaction obtained in the previous section. The fact that it depends on $u_{\mathrm{S}}$ is a consequence of that interaction being state dependent (as is usual for effective interactions), and $u_{\mathrm{S}}$ should henceforth be considered just as an external mechanism used to tune the interaction. Consequently all that we can derive from the free-energy functional of a system can also be derived from this functional for the effective fluid.

Let us separate out the ideal and excess parts:

$$
\begin{aligned}
& F_{\text {eff }}\left[u_{\mathrm{S}}, \rho\right]=k T \int \mathrm{d} \boldsymbol{r} \rho(\boldsymbol{r})\left[\ln \mathcal{V}_{\mathrm{L}} \rho(\boldsymbol{r})-1\right]+F_{\mathrm{eff}}^{\mathrm{ex}}\left[u_{\mathrm{S}}, \rho\right] \\
& F_{\mathrm{eff}}^{\mathrm{ex}}\left[u_{\mathrm{S}}, \rho\right] \equiv k T \int \mathrm{d} \boldsymbol{r} \rho_{\mathrm{S}}(\boldsymbol{r})\left[\ln z^{-1} \rho_{\mathrm{S}}(\boldsymbol{r})-1\right]+F^{\mathrm{ex}}\left[\rho, \rho_{\mathrm{S}}\right]-\Upsilon_{0}\left[u_{\mathrm{S}}\right]
\end{aligned}
$$

with $\rho_{\mathrm{S}}$ given by equation (6) (setting $\rho_{\mathrm{L}}=\rho$ ).

We can now obtain the DCF of the effective fluid as

$$
c_{\mathrm{eff}}\left(\boldsymbol{r}, \boldsymbol{r}^{\prime}\right)=-\beta \frac{\delta^{2} F_{\mathrm{eff}}^{\mathrm{ex}}}{\delta \rho(\boldsymbol{r}) \delta \rho\left(\boldsymbol{r}^{\prime}\right)} .
$$

The first derivative yields (notice that (6) implies $\delta F_{\text {eff }}^{\mathrm{ex}} / \delta \rho_{\mathrm{S}}(\boldsymbol{r})=0$ )

$$
\frac{\delta F_{\mathrm{eff}}^{\mathrm{ex}}}{\delta \rho(\boldsymbol{r})}=-\omega_{1}+\frac{\delta F^{\mathrm{ex}}}{\delta \rho(\boldsymbol{r})}
$$


and further differentiation leads to

$$
\frac{\delta^{2} F_{\mathrm{eff}}^{\mathrm{ex}}}{\delta \rho(\boldsymbol{r}) \delta \rho\left(\boldsymbol{r}^{\prime}\right)}=\frac{\delta^{2} F^{\mathrm{ex}}}{\delta \rho(\boldsymbol{r}) \delta \rho\left(\boldsymbol{r}^{\prime}\right)}+\int \mathrm{d} \boldsymbol{r}^{\prime \prime} \frac{\delta^{2} F^{\mathrm{ex}}}{\delta \rho(\boldsymbol{r}) \delta \rho_{\mathrm{S}}\left(\boldsymbol{r}^{\prime \prime}\right)} \frac{\delta \rho_{\mathrm{S}}\left(\boldsymbol{r}^{\prime \prime}\right)}{\delta \rho\left(\boldsymbol{r}^{\prime}\right)} .
$$

Therefore (10) and the DCF matrix definition for the mixture

$$
c_{i j}\left(\boldsymbol{r}, \boldsymbol{r}^{\prime}\right)=-\beta \frac{\delta^{2} F^{\mathrm{ex}}}{\delta \rho_{i}(\boldsymbol{r}) \delta \rho_{j}\left(\boldsymbol{r}^{\prime}\right)}
$$

are related by

$$
c_{\mathrm{eff}}\left(\boldsymbol{r}, \boldsymbol{r}^{\prime}\right)=c_{\mathrm{LL}}\left(\boldsymbol{r}, \boldsymbol{r}^{\prime}\right)+\int \mathrm{d} \boldsymbol{r}^{\prime \prime} c_{\mathrm{LS}}\left(\boldsymbol{r}, \boldsymbol{r}^{\prime \prime}\right) \frac{\delta \rho_{\mathrm{S}}\left(\boldsymbol{r}^{\prime \prime}\right)}{\delta \rho\left(\boldsymbol{r}^{\prime}\right)} .
$$

By differentiating equation (6) with respect to $\rho\left(r^{\prime}\right)$ we obtain

$$
\int \mathrm{d} \boldsymbol{r}^{\prime \prime}\left[\frac{1}{\rho_{\mathrm{S}}(\boldsymbol{r})} \delta\left(\boldsymbol{r}-\boldsymbol{r}^{\prime \prime}\right)-c_{\mathrm{SS}}\left(\boldsymbol{r}, \boldsymbol{r}^{\prime \prime}\right)\right] \frac{\delta \rho_{\mathrm{S}}\left(\boldsymbol{r}^{\prime \prime}\right)}{\delta \rho\left(\boldsymbol{r}^{\prime}\right)}=c_{\mathrm{SL}}\left(\boldsymbol{r}, \boldsymbol{r}^{\prime}\right) .
$$

In principle equations (11) and (12) permit us to eliminate $\delta \rho_{\mathrm{S}}\left(\boldsymbol{r}^{\prime \prime}\right) / \delta \rho\left(\boldsymbol{r}^{\prime}\right)$ and obtain the functional $c_{\text {eff }}\left(\boldsymbol{r}, \boldsymbol{r}^{\prime}\right)$ in terms of the matrix functional $c_{i j}\left(\boldsymbol{r}, \boldsymbol{r}^{\prime}\right)$. In practice this is a difficult task except for very special cases (which we will deal with a bit later). In spite of that it is always possible to achieve it as a functional expansion in 'powers' of $\rho_{\mathrm{S}}$. To show this let us rewrite (12) as

By defining

$$
\frac{\delta \rho_{\mathrm{S}}\left(\boldsymbol{r}^{\prime \prime}\right)}{\delta \rho\left(\boldsymbol{r}^{\prime}\right)}=\rho_{\mathrm{S}}\left(\boldsymbol{r}^{\prime \prime}\right) c_{\mathrm{SL}}\left(\boldsymbol{r}^{\prime \prime}, \boldsymbol{r}^{\prime}\right)+\rho_{\mathrm{S}}\left(\boldsymbol{r}^{\prime \prime}\right) \int \mathrm{d} \boldsymbol{r}^{\prime \prime \prime} c_{\mathrm{SS}}\left(\boldsymbol{r}^{\prime \prime}, \boldsymbol{r}^{\prime \prime \prime}\right) \frac{\delta \rho_{\mathrm{S}}\left(\boldsymbol{r}^{\prime \prime \prime}\right)}{\delta \rho\left(\boldsymbol{r}^{\prime}\right)}
$$

$$
(a * b)\left(\boldsymbol{r}, \boldsymbol{r}^{\prime}\right) \equiv \int \mathrm{d} \boldsymbol{r}^{\prime \prime} a\left(\boldsymbol{r}, \boldsymbol{r}^{\prime \prime}\right) \rho_{\mathrm{S}}\left(\boldsymbol{r}^{\prime \prime}\right) b\left(\boldsymbol{r}^{\prime \prime}, \boldsymbol{r}^{\prime}\right)
$$

equation (11) can be expressed as

$$
c_{\mathrm{eff}}\left(\boldsymbol{r}, \boldsymbol{r}^{\prime}\right)=c_{\mathrm{LL}}\left(\boldsymbol{r}, \boldsymbol{r}^{\prime}\right)+\sum_{n=0}^{\infty}(c_{\mathrm{LS}} * \overbrace{c_{\mathrm{SS}} * \cdots * c_{\mathrm{SS}}}^{n \text { times }} * c_{\mathrm{SL}})\left(\boldsymbol{r}, \boldsymbol{r}^{\prime}\right) .
$$

Equation (13) is very 'expressive' as regards how depletion contributes to the direct correlations of the effective-fluid particles: apart from the direct correlation between two large particles, this can also be mediated by one, two, etc, small particles.

These expressions have full generality: they are valid for any binary mixture of any kind and in any particular phase. But the equations can be more compactly written if we particularize to the uniform fluid. In this case the density profiles are constant and all the DCFs depend on the difference of their arguments. Then, Fourier transforming equations (11) and (12) (or alternatively equation (13)) we can simply write

$$
\hat{c}_{\mathrm{eff}}(\boldsymbol{k})=\hat{c}_{\mathrm{LL}}(\boldsymbol{k})+\frac{\rho_{\mathrm{S}} \hat{c}_{\mathrm{LS}}(\boldsymbol{k})^{2}}{1-\rho_{\mathrm{S}} \hat{c}_{\mathrm{SS}}(\boldsymbol{k})} .
$$

There is an alternative interpretation of equation (14); it becomes clear if we compute the structure factor of the effective fluid, namely

$$
S_{\mathrm{eff}}(\boldsymbol{k})=\frac{1}{1-\rho_{\mathrm{L}} \hat{c}_{\mathrm{eff}}(\boldsymbol{k})}=\frac{1-\rho_{\mathrm{S}} \hat{c}_{\mathrm{SS}}(\boldsymbol{k})}{\operatorname{det}\left[\delta_{i j}-\rho_{i} \hat{c}_{i j}(\boldsymbol{k})\right]}=S_{\mathrm{LL}}(\boldsymbol{k}) .
$$

In other words, the structure factor of the effective fluid is simply the element of the structurefactor matrix corresponding to correlations between large particles in the mixtures. Actually this was the starting point from which the effective fluid was defined in reference [12]. Seen in this light, it appears as the result of the semi-grand ensemble choice, and equations (11) and (12) extend it to arbitrary phases. 


\subsection{Application to approximate density functional theories}

The usefulness of equations (9) and (14) is that they are the basic ingredients for carrying out any of the standard approximations of DFT [11]. The direct way to deal with a fluid of hard spheres plus an attractive well is to use an approximate DFT to describe the hard spheres and then incorporate the attraction perturbatively [10,13-16]. This can be done with the depletion potential (obtained by any valid procedure [3]), and the results map out the phase diagram of very asymmetric binary hard-sphere mixtures [7].

There are rather good approximations for the free energy of additive mixtures of hard spheres, and that is the only requisite for obtaining the free energy (9) for the effective fluid. Then equation (14) provides its DCF, so we can directly apply one of the standard approximate density functionals without resorting to the perturbative method. We are at present trying to test this method on the very asymmetric binary mixture of hard spheres.

\section{The limit of infinite asymmetry}

Another possibility offered by the functional form expressed in equations (6), (8), and (9) is that of studying the 'infinite-asymmetry' limit, i.e. the limit in which the size of the small particles goes to zero while the effect of depletion is retained. This limit was already used [12] to show that the DCF of a mixture of hard spheres becomes that of a fluid of adhesive hard spheres in this limit. The possibility offered by our formalism is that of taking the limit on the functional to obtain a limiting functional for the effective fluid.

Although not conceptually difficult, this limit is rather involved; thus instead of taking it for a system of hard spheres we have chosen the simpler system of parallel hard cubes. The reason for this simplicity is that the effect of depletion is stronger for cubes than for spheres (see figure 1). This is reflected in the fact that the small-spheres packing fraction has to be $\mathrm{O}(1)$ to maintain the effect of depletion, whereas the small-cubes packing fraction has to be $\mathrm{O}(\epsilon)$ ( $\epsilon$ being the small-to-large edge-length ratio) to produce the same effect (again the reason becomes apparent in figure 1). Parallel hard cubes constitute a rather academic model, which can only be justified because of its simpler treatment. Nevertheless, although it presents important differences with respect to a system of hard spheres (e.g. freezing is a continuous transition, at freezing the solid has a large amount of vacancies, etc; see reference [8] for more details on the phase behaviour of this system), the qualitative behaviours of the two mixtures have many similarities.

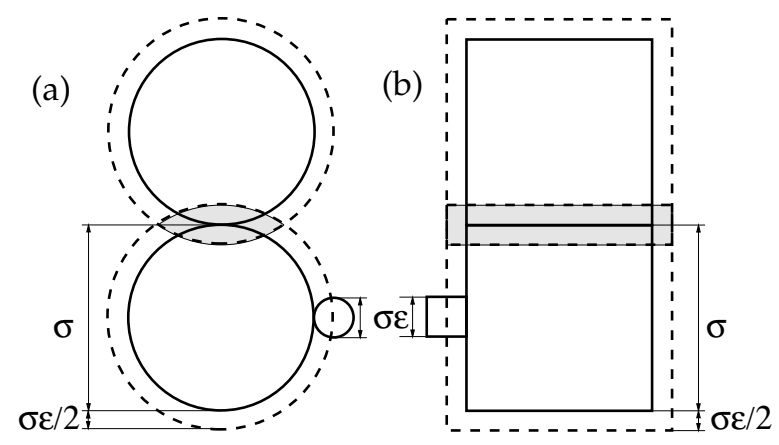

Figure 1. The increment in the total volume, $\Delta V$, available to the small particles when two large particles come to touch each other (shaded region). If $v_{0}$ denotes the volume of a large particle, $\Delta v$ is (a) $v_{0} \epsilon^{2}(3 / 2+\epsilon)$ for hard spheres, and (b) $v_{0} \epsilon(1+\epsilon)^{2}$ for parallel hard cubes. 


\subsection{The fundamental-measure functional for mixtures of parallel hard cubes}

In principle the limit can be taken for any available functional (even 'classical' ones); however, one would expect better results from those able to deal with substantial inhomogeneities (a fluid of small particles in the interstices of a lattice of big ones can be considered as a kind of confined fluid). In recent work [17] we have proposed a density functional for a mixture of parallel hard cubes based on the so-called fundamental-measure theory $[11,18]$. This theory has been shown to produce functionals very suitable for dealing with substantial inhomogeneities $[17,19,20]$, and in its construction one does not need to know the DCF of the fluid (as a matter of fact, it can be derived from the functional afterwards). The details of the construction can be found elsewhere [17], but the final form of the functional is rather simple. If we define the weights (or 'fundamental measures')

$$
\begin{aligned}
\omega_{i}^{(3)}(\boldsymbol{r}) & \equiv \Theta\left(\sigma_{i}^{x} / 2-|x|\right) \Theta\left(\sigma_{i}^{y} / 2-|y|\right) \Theta\left(\sigma_{i}^{z} / 2-|z|\right) \\
\boldsymbol{\omega}_{i}^{(2)}(\boldsymbol{r}) & \equiv\left(\frac{\partial}{\partial \sigma_{i}^{x}}, \frac{\partial}{\partial \sigma_{i}^{y}}, \frac{\partial}{\partial \sigma_{i}^{z}}\right) \omega_{i}^{(3)}(\boldsymbol{r}) \\
\boldsymbol{\omega}_{i}^{(1)}(\boldsymbol{r}) & \equiv\left(\frac{\partial^{2}}{\partial \sigma_{i}^{y} \partial \sigma_{i}^{z}}, \frac{\partial^{2}}{\partial \sigma_{i}^{z} \partial \sigma_{i}^{x}}, \frac{\partial^{2}}{\partial \sigma_{i}^{x} \partial \sigma_{i}^{y}}\right) \omega_{i}^{(3)}(\boldsymbol{r}) \\
\omega_{i}^{(2)}(\boldsymbol{r}) & \equiv \omega_{i}^{(2, x)}(\boldsymbol{r})+\omega_{i}^{(2, y)}(\boldsymbol{r})+\omega_{i}^{(2, z)}(\boldsymbol{r}) \\
\omega_{i}^{(1)}(\boldsymbol{r}) & \equiv \omega_{i}^{(1, x)}(\boldsymbol{r})+\omega_{i}^{(1, y)}(\boldsymbol{r})+\omega_{i}^{(1, z)}(\boldsymbol{r}) \\
\omega_{i}^{(0)}(\boldsymbol{r}) & \equiv \frac{\partial^{3}}{\partial \sigma_{i}^{x} \partial \sigma_{i}^{y} \partial \sigma_{i}^{z}} \omega_{i}^{(3)}(\boldsymbol{r})
\end{aligned}
$$

where $\sigma_{i}^{\mu}$ is the edge length along the $\mu$-axis of species $i$, then in terms of the weighted densities

$$
n_{\mu}=\sum_{i} \rho_{i} * \omega_{i}^{(\mu)} \quad \mu=0,1,2,3
$$

$\left(n_{\mu}\right.$ inherits the scalar/vector character of the corresponding weight), the functional has the form

$F^{\mathrm{ex}}\left[\left\{\rho_{i}\right\}\right]=\int \mathrm{d} \boldsymbol{r} \Phi\left(\left\{n_{\mu}(\boldsymbol{r})\right\}\right) \quad \Phi=-n_{0} \ln \left(1-n_{3}\right)+\frac{\boldsymbol{n}_{1} \cdot \boldsymbol{n}_{2}}{1-n_{3}}+\frac{n_{2, x} n_{2, y} n_{2, z}}{\left(1-n_{3}\right)^{2}}$.

Clearly this functional is valid for a mixture of aligned parallelepipeds, but we will only consider here the case of cubes $\left(\sigma_{i}^{x}=\sigma_{i}^{y}=\sigma_{i}^{z}=\sigma_{i}\right)$.

Let us henceforth consider a binary mixture of parallel hard cubes with edge lengths $\sigma_{\mathrm{L}}=1$ and $\sigma_{\mathrm{S}}=\epsilon$. According to (18), the DCF of this fluid will be [17]

$$
c_{i j}(\boldsymbol{r})=\left[\chi_{0}+\chi_{1} R_{i j}(\boldsymbol{r})+\chi_{2} S_{i j}(\boldsymbol{r})+\chi_{3} V_{i j}(\boldsymbol{r})\right] f_{i j}(\boldsymbol{r})
$$

where

$$
\begin{aligned}
& f_{i j}=-\omega_{i}^{(3)} * \omega_{j}^{(0)}-\omega_{i}^{(0)} * \omega_{j}^{(3)}-\omega_{i}^{(2)} * \omega_{j}^{(1)}-\omega^{(1)} * \omega^{(2)} \\
& R_{i j}=\omega_{i}^{(3)} * \omega_{j}^{(1)}+\omega_{i}^{(1)} * \omega_{j}^{(3)}+\omega_{i}^{(2)} * \omega_{j}^{(2)}-\omega_{i}^{(2)} * \omega_{j}^{(2)} \\
& S_{i j}=\omega_{i}^{(3)} * \omega_{j}^{(2)}+\omega_{i}^{(2)} * \omega_{j}^{(3)} \\
& V_{i j}=\omega_{i}^{(3)} * \omega_{j}^{(3)}
\end{aligned}
$$


(a dot product is implied in the convolutions of vector weights; $f_{i j}$ is simply the Mayer function of cubes $i$ and $j$ ) and

$$
\begin{aligned}
\chi_{0} & =\frac{1}{1-\xi_{3}} & \chi_{2} & =\frac{\xi_{1}}{\left(1-\xi_{3}\right)^{2}}+\frac{2 \xi_{2}^{2}}{\left(1-\xi_{3}\right)^{3}} \\
\chi_{1} & =\frac{\xi_{2}}{\left(1-\xi_{3}\right)^{2}} & \chi_{3} & =\frac{\xi_{0}}{\left(1-\xi_{3}\right)^{2}}+\frac{6 \xi_{1} \xi_{2}}{\left(1-\xi_{3}\right)^{3}}+\frac{6 \xi_{2}^{3}}{\left(1-\xi_{3}\right)^{4}}
\end{aligned}
$$

with $\xi_{k} \equiv \rho_{\mathrm{L}}+\epsilon^{k} \rho_{\mathrm{S}}$.

\subsection{The effective fluid in the limit of infinite asymmetry}

We can now use equation (14) to compute the DCF of the effective fluid, and in doing so (after some tedious algebra) one realizes that in the $\epsilon \rightarrow 0$ limit (infinite asymmetry) $c_{\text {eff }}$ goes to a finite non-trivial limit provided that we choose $\epsilon^{2} \rho_{\mathrm{S}}=\mathrm{O}(1)$ (as expected from figure 1). The limit can be simply written as

$$
c_{\text {eff }}(\boldsymbol{r})=c_{\mathrm{PHC}}(\boldsymbol{r})+c_{\mathrm{ad}}(\boldsymbol{r})
$$

where $c_{\mathrm{PHC}}$ is $c_{\mathrm{LL}}$ for $\xi_{k}=\rho_{\mathrm{L}}(k=0,1,2,3)$, i.e. the DCF of the one-component fluid of large cubes, and

$c_{\mathrm{ad}}(\boldsymbol{r})=-\frac{z}{2\left(1-\rho_{\mathrm{L}}\right)}\left\{\delta_{\mathrm{S}}(\boldsymbol{r})+\frac{\rho_{\mathrm{L}}}{1-\rho_{\mathrm{L}}} S_{\mathrm{LL}}(\boldsymbol{r})+\frac{6 \rho_{\mathrm{L}}^{2}}{\left(1-\rho_{\mathrm{L}}\right)^{2}} V_{\mathrm{LL}}(\boldsymbol{r})\right\} f_{\mathrm{LL}}(\boldsymbol{r})$

where $z=\epsilon^{2} \exp \left(\beta \mu_{\mathrm{S}}\right) / \mathcal{V}_{\mathrm{S}}$, the rescaled fugacity of the small cubes, and

$$
\begin{aligned}
\delta_{\mathrm{S}}(\boldsymbol{r})=\delta(1-|x|)(1-|y|)(1-|z|) & \\
& +(1-|x|) \delta(1-|y|)(1-|z|)+(1-|x|)(1-|y|) \delta(1-|z|)
\end{aligned}
$$

is a surface adhesion term. It is precisely the latter that relates this result to the one obtained for hard spheres in reference [12]. Therefore the effective fluid can be referred to as the fluid of parallel adhesive hard cubes.

But we can go beyond this, by taking the limit in the functional $F_{\text {eff }}$ itself. As we now know the scaling of the small-particles density, let us define

$$
\xi(\boldsymbol{r}) \equiv \epsilon^{2} \rho_{\mathrm{S}}(\boldsymbol{r})
$$

in terms of which the equilibrium equation (6) can be written as

$$
\ln (\xi / z)=-\sum_{\alpha} \frac{\partial \Phi}{\partial n_{\alpha}} * \omega_{\mathrm{S}}^{(\alpha)} .
$$

Now, for a given $f(\boldsymbol{r})$,

$$
\begin{aligned}
& f * \omega_{\mathrm{S}}^{(\alpha)}=\epsilon^{\alpha} f+\mathrm{O}\left(\epsilon^{\alpha+2}\right) \quad \alpha \neq 0 \\
& f * \omega_{\mathrm{S}}^{(0)}=f+\frac{\epsilon^{2}}{8} \nabla^{2} f+\mathrm{O}\left(\epsilon^{4}\right)
\end{aligned}
$$

when $\epsilon \rightarrow 0$; therefore, assuming a power expansion in $\epsilon$ for $\xi(\boldsymbol{r})$, equation (22) yields

$$
\xi=z\left(1-\bar{n}_{3}\right)\left\{1-\epsilon\left(8 z+\frac{\bar{n}_{2}}{1-\bar{n}_{3}}\right)+\epsilon^{2} \xi_{2}+\mathrm{O}\left(\epsilon^{3}\right)\right\}
$$

where the overline denotes the corresponding weighted densities for the one-component largecube fluid. The actual expression of $\xi_{2}$ is absolutely irrelevant in what follows. This equation eliminates the density of the small cubes in terms of that of the large ones and $z$. (Notice that 
usually this cannot be done explicitly, but in this case it is feasible because we are taking the $\epsilon \rightarrow 0$ limit.) Substituting $\xi$ in (18) and using again the expansions (23) we finally obtain

$$
F_{\text {eff }}[\rho]=F_{\mathrm{PHC}}[\rho]+F_{\text {ad }}[\rho]+\mathrm{O}(\epsilon)
$$

where $F_{\mathrm{PHC}}$ is the free-energy functional for the one-component fluid of (large) parallel hard cubes, and $F_{\text {ad }}$ is an 'adhesive' contribution arising from depletion. Its expression in terms of weighted densities is

$$
F_{\mathrm{ad}}[\rho]=\int \mathrm{d} \boldsymbol{r} \Phi_{\mathrm{ad}}\left(\left\{\bar{n}_{\alpha}\right\}\right) \quad \Phi_{\mathrm{ad}}=\frac{z}{8} \frac{\left|\nabla \bar{n}_{3}\right|^{2}-4 \overline{\boldsymbol{n}}_{2} \cdot \overline{\boldsymbol{n}}_{2}}{1-\bar{n}_{3}} .
$$

\subsection{Phase behaviour of the effective fluid}

It is straightforward to derive the equation of state for a uniform effective fluid; its expression is

$$
\beta p=y+3(1-z / 2) y^{2}+2 y^{3} \quad y \equiv \rho_{\mathrm{L}} /\left(1-\rho_{\mathrm{L}}\right) .
$$

It has a van der Waals loop, with critical point $z^{\mathrm{c}}=2+\sqrt{8 / 3}, \rho_{\mathrm{L}}^{\mathrm{c}}=1 /(1+\sqrt{6})$, corresponding to a fluid-fluid demixing of the large and small cubes.

But it is not difficult to realize that $F_{\text {ad }} \rightarrow-\infty$ as $\rho(\boldsymbol{r}) \rightarrow \sum_{\boldsymbol{R}} \delta(\boldsymbol{r}-\boldsymbol{R})$, with the $\boldsymbol{R}$ the sites of a close-packed simple cubic lattice. This means that the only stable phase of this fluid at any state point is the close-packed solid. It turns out that this 'collapse' is a pathology not exclusive to this model: Stell proved [21] that for a system of adhesive hard spheres there is no well-defined partition function, and simulations of square-well fluids show [9] that as the range of the well goes to zero while the second virial coefficient is maintained (the adhesive limit), the coexistence curve 'eats up' the whole phase diagram and the two limiting coexisting phases are a 'zero-density' fluid and a close-packed solid.

Nevertheless our functional (25), (26) tells us more than that. It shows that there is a rich metastable phase diagram in which we have fluid and solid phases separated from the close packing by high free-energy barriers. This phase behaviour is illustrated in figure 2 .

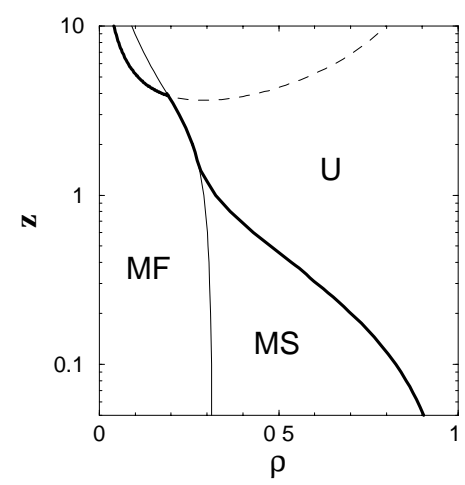

Figure 2. Solvent fugacity, $z$, versus solute packing fraction, $\rho$, for the infinitely asymmetric binary mixture of parallel hard cubes. The thick solid line separates the unstable region (U) from the metastable one; the thin one marks the (continuous) transition from a metastable fluid (MF) to a metastable solid (MS); the dashed one is the fluid-fluid spinodal.

According to this diagram, the typical situation is as follows. At large values of $z$ the fluid will quickly collapse into the close-packed configuration. At small values of $z$, however, the fluid will be trapped in metastable phases for very long times; upon increasing $\rho$ the fluid will 
be seen to undergo a continuous fluid-solid transition at $\rho \approx 0.3$, and it can remain solid for a very long period of time; if we move to a still higher $\rho$ the system will eventually collapse, but as it may take a long time for the whole system to do this, there may be an apparent expanded-solid-collapsed-solid coexistence. This picture is a caricature of the typical phase diagram of a fluid exhibiting an isostructural solid-solid transition $[9,10]$.

\subsection{An effective fluid with a small polydispersity}

It has been suggested that the existence of a small amount of polydispersity in the adhesive hard spheres may regularize the partition function and thus avoid the collapse and induce a non-trivial phase behaviour $[9,21]$. This is true for the present system within the fundamentalmeasure approximation [8]. To show this, we have assumed that the large cubes are now polydisperse, and have introduced polydispersity in the simplest possible way: cubes are replaced by parallelepipeds with their three edge lengths randomly chosen from a Gaussian distribution with mean 1 and variance $\Delta \sigma^{2}$. With this choice the free energy of the uniform fluid (and hence the fluid-fluid coexistence) is still given by (27). If we take $\Delta \sigma \ll 1$ we can simply ignore the Gaussian tail in the negative lengths, and, more importantly, also a possible fractionation into solids with different compositions at very large packing fractions. In fact, we expect that $\rho_{\mathrm{L}}(\boldsymbol{r} ; \boldsymbol{\sigma})=\rho_{\mathrm{L}}(\boldsymbol{r}) \mathcal{P}(\boldsymbol{\sigma})$, with $\mathcal{P}$ the probability density of having edge lengths $\boldsymbol{\sigma}=\left(\sigma_{x}, \sigma_{y}, \sigma_{z}\right)$. In the fundamental-measure formalism this implies that the weights $\omega_{\mathrm{L}}^{(\alpha)}$ must be replaced by their averages $\widetilde{\omega}_{\mathrm{L}}^{(\alpha)}$ over $\mathcal{P}$, which are like 'smoothed' versions of the former ones (for the Gaussian choice we have made they can be computed analytically [8]). This completely eliminates the divergence of $\Phi_{\mathrm{ad}}$, no matter how small $\Delta \sigma$ is.

In figure 3 we have plotted the phase diagrams of the effective fluid for three different choices of $\Delta \sigma$. In all three the phase behaviours are similar: a region of coexistence between a low-density fluid and a nearly close-packed solid (large $z$ ); a region in which a continuous freezing transition is followed, at higher packing fractions, by a first-order isostructural solidsolid transition (medium $z$ ); and finally a region, below the critical point, in which there is only a freezing transition (small $z$ ). In all cases the fluid-fluid transition is metastable. As $\Delta \sigma \rightarrow 0$ the coexistence line 'eats up' the phase diagram, as expected; however, these phase
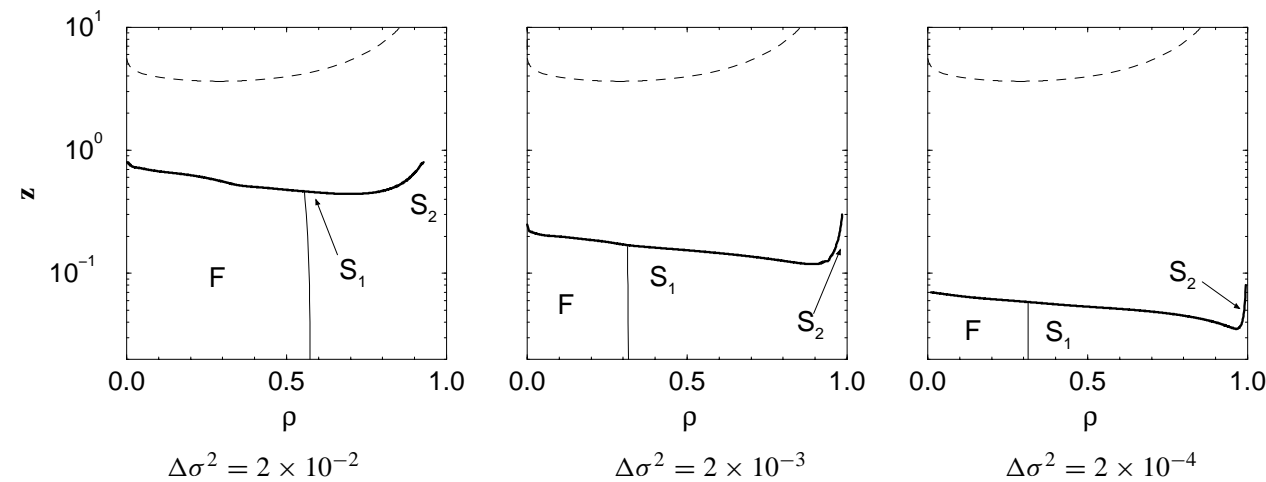

Figure 3. Rescaled solvent fugacity, $z$, versus solute packing fraction, $\rho$, for the effective polydisperse fluid for three values of the standard deviation of the variance $\Delta \sigma^{2}$. In each panel, the thick solid line marks the fluid-solid or solid-solid coexistence (notice that coexistence extends up to higher values of $z$, although lines are not continued for numerical reasons); the thin one marks the continuous fluid-solid transition below the coexistence region; the dashed line is the metastable fluid-fluid coexistence (notice that it is independent of $\Delta \sigma$ ). 
diagrams shed light on the metastable phase behaviour of the monodisperse case.

In terms of the very asymmetric binary mixture, the present phase diagram shows that, in general (for sufficiently large $z$ ), fluid-fluid demixing is largely pre-empted by fluid-solid demixing (i.e. the phase rich in large particles is a highly packed solid). However, there is a range of $z$ in which the large particles first freeze and it is only at higher densities that demixing occurs, separating the system into two solid phases, one of them very highly packed (just as before). Below a critical $z$, no more demixing occurs, although freezing still shows up.

\section{Conclusions}

In the last two years evidence has been accumulating supporting this demixing scenario for very asymmetric additive binary mixtures of hard particles [5-7,22], a behaviour already suggested by experiments $[23,24]$. The remarkable feature is the feasibility of studying the phase behaviour of a mixture via an effective one-component fluid interacting through the depletion interaction. This is in fact why the qualitative behaviour of this system is similar to that of colloids with narrow and deep attractive wells (adhesive-like interactions). We have shown here that, in spite of its singularity, the adhesive limit is the key to understanding these mixtures when the asymmetry is very large.

Our major contribution to this subject is more density functional oriented. On one hand, we have introduced in section 2.2 a promising formalism for mapping the DCF and free energy of a mixture onto that of an effective fluid, so we have all the necessary ingredients to use one of the 'classical' functionals (of the 'weighted-density' or 'effective-fluid' type). We are at present testing this method. On the other hand, we have shown that fundamental-measure theories are suitable for studying these systems in the limit of infinitely large asymmetry. This viewpoint may be very useful when applied to other systems and other transitions.

\section{Acknowledgment}

JAC's work is part of the project PB96-0119 of the Dirección General de Enseñanza Superior (Spain).

\section{References}

[1] Biben T and Hansen J-P 1991 Phys. Rev. Lett. 662215

[2] Asakura S and Oosawa F 1954 J. Chem. Phys. 221255

[3] Götzelmann B, Evans R and Dietrich S 1998 Phys. Rev. E 576785 Götzelmann B, Roth R, Dietrich S, Dijkstra M and Evans R 1999 Europhys. Lett. 47398

[4] Biben T, Bladon P and Frenkel D 1996 J. Phys. Condens. Matter 810799

[5] Dijkstra M, van Roij R and Evans R 1998 Phys. Rev. Lett. 812268 Dijkstra M, van Roij R and Evans R 1999 Phys. Rev. Lett. 82117 Dijkstra M, van Roij R and Evans R 1999 Phys. Rev. E 595744

[6] Almarza N G and Enciso E 1999 Phys. Rev. E 594426

[7] Velasco E, Navascués G and Mederos L 1999 Phys. Rev. E 603158

[8] Martínez-Ratón Y and Cuesta J A 1998 Phys. Rev. E 58 R4080 Martínez-Ratón Y and Cuesta J A 1999 J. Chem. Phys. 111317

[9] Bolhuis P and Frenkel D 1994 Phys. Rev. Lett. 722211 Bolhuis P, Haagen M and Frenkel D 1994 Phys. Rev. E 504880

[10] Tejero C F, Daanoun A, Lekkerkerker H N W and Baus M 1994 Phys. Rev. Lett. 73752 Tejero C F, Daanoun A, Lekkerkerker H N W and Baus M 1995 Phys. Rev. E 51558

[11] Evans R 1992 Fundamentals of Inhomogeneous Fluids ed D Henderson (New York: Dekker) p 85

[12] Heno Y and Regnaut C 1991 J. Chem. Phys. 959204 
[13] Likos C, Németh Zs T and Löwen H 1994 J. Phys. Condens. Matter 610965

[14] Rascón C, Mederos L and Navascués G 1996 Phys. Rev. Lett. 772249

[15] Kim S-C and Suh S-H 1997 Phys. Rev. E 562889

[16] Denton A R and Löwen H 1997 J. Phys. Condens. Matter 9 L1

[17] Cuesta J A and Martínez-Ratón Y 1997 Phys. Rev. Lett. 783681

Cuesta J A and Martínez-Ratón Y 1997 J. Chem. Phys. 1076379

[18] Rosenfeld Y 1989 Phys. Rev. Lett. 69980

Note the sign error discussed in

Phan S, Kierlik E, Rosinberg M L, Bildstein B and Kahl G 1993 Phys. Rev. E 48618

[19] Rosenfeld Y, Schmidt M, Löwen H and Tarazona P 1997 J. Phys. Condens. Matter 8 L577

Rosenfeld Y, Schmidt M, Löwen H and Tarazona P 1997 Phys. Rev. E 554245

[20] Tarazona P and Rosenfeld Y 1997 Phys. Rev. E 55 R4873

[21] Stell G 1991 J. Stat. Phys. 631203

[22] Buhot A and Krauth W 1999 Phys. Rev. E 592939

[23] Imhof A and Dhont J K G 1995 Phys. Rev. Lett. 751662

[24] Dinsmore A D, Yodh A G and Pine D J 1995 Phys. Rev. E 524045 